\section{Virtual Reality-Based Orthopedic Telerehabilitation}

\author{
Grigore Burdea, Viorel Popescu, Vincent Hentz, and Kerri Colbert
}

\begin{abstract}
Rehabilitation interventions in remote areas are problematic because of distance and available resources. Orthopedic impairments acquired by individuals in remote areas can then lead to permanent disabilities/loss of function because of lack of appropriate rehabilitation. A system being developed by Rutgers and Stanford Universities provides therapy at the patient's home, with remote monitoring and periodic re-assessment. This telerehabilitation system uses virtual reality and haptic interfaces, and a pair of networked PCs. It is intended for rehabilitation of patients with hand, elbow, knee and ankle impairements. Data from the first patient treated with the telerehabilitation system is encouraging.
\end{abstract}

Index Terms-Haptic interfaces, orthopedic rehabilitation, remote evaluation, Rutgers Master, virtual reality exercises.

\section{INTRODUCTION}

The number of patients needing rehabilitation (including long-term therapy) has increased in recent years [1], but available resources have unfortunately diminished. The reduction in covered duration of therapy, and the lack of timely interventions can lead to permanent disabilities in otherwise reversible conditions.

Timeliness and duration of rehabilitative therapy are especially problematic for those in remote rural locations or living in depressed urban areas. In such instances there are no clinics in the vicinity of the patient's home. Patients with orthopedic impairments, such as those that have had hand or knee surgery, typically follow a regimen of combined clinic and home rehabilitation exercises. Home exercises are currently done on simple mechanical systems that are loaned to the patient. Since these mechanical devices are not networked, there is no way a therapist can either monitor patient's progress at a distance, or change exercise effort (difficulty) levels from the clinic. There is also no way to verify that indeed the patient has done the prescribed home rehabilitation exercises, and some patients feel less motivated to exercise at home without direct medical supervision.

A solution to the above problem would be to provide rehabilitation therapy at the patient's home, without sacrificing quality of care. Remote monitoring of patient's progress needs to be present, preferably using a networked computerized system (telerehabilitation). Such remote therapeutic intervention will clearly increase the disabled individual's self sufficiency, as well as family support and involvement in the therapy. For disabled individuals that are self-employed, or perform home-based employment on a regular basis, telerehabilitation will be beneficial through the elimination of the time needed to travel to and from the clinic. For individuals on temporary disability, such a system would also shorten time lost from work, because of an intensive and monitored home-based therapeutic intervention. In the case of orthopedic patients, special force feedback interfaces need to be developed to allow on-line rehabilitation at home. Clinic-based computerized evaluation has been used for a decade [7], [9], [10], [6],

Manuscript received June 23, 1999; revised November 5, 1999 and February 25,2000 . This work was supported by grants from the National Science Foundation (BES-9708020) and from the Rutgers University (Special Research Opportunity Award).

G. Burdea and V. Popescu are with the CAIP Center, Rutgers-The State University of New Jersey, Piscataway, NJ 08854 USA http://www.caip.rutgers.edu/vrlab.

V. R. Hentz and K. Colbert are with Division of Hand and Upper Extremity Surgery, School of Medicine, Stanford University, 900 Welch Rd. \#15, Stanford, CA 94304 USA.

Publisher Item Identifier S 1063-6528(00)07296-7. but the systems are for evaluation only, not for therapy, and are not networked.

Providing home-based therapy without home care therapists should reduce health care costs, while maintaining quality. In this era of medical cost consciousness, the concept of "budget neutrality" has become a major factor in cost-benefit analysis of a new treatment or an extension of a current treatment to a new problem. This philosophy states that the cost of implementing any new technology, drug, etc., must be offset by a comparable savings to the global cost of medical care in terms of reduced long term care costs, lowered risk of the need for later more expensive care, etc. The clinical implementation of a telerehabilitation system will clearly be associated with increased initial costs including the obvious cost of hardware, software and therapist training.

This communication describes a virtual reality-based telerehabilitation system being developed jointly by Rutgers and Stanford Universities. Section II presents the system components, while Section III details the library of rehabilitation exercises, and includes clinical data from the first patient trial. Section IV concludes this paper.

\section{SYSTEM COMPONENTS}

The prototype of the orthopedic telerehabilitation system consists of a pair of similar PCs (one at the patient's home and the other at the clinic), connected over the Internet (see Fig. 1). The home rehabilitation station in turn consists of a force feedback glove called the "Rutgers Master," [2] a multipurpose control interface connected to the PC, a net camera, and a microphone array [13]. The sensing glove measures finger grasping and abduction/adduction motion and sustains resistive forces up to $16 \mathrm{~N}$ at each fingertip. It weighs only 100 grams due to the use of pneumatic actuators. These are custom glass-graphite pistons that have extremely low friction and high dynamic range. The actuators can sustain high forces without overheating and damage.

The multipurpose control interface has an embedded Pentium board (250 MHz), two pneumatic controllers and custom electronics to read glove sensors and control its feedback actuators [14]. The pneumatic controllers are $500 \mathrm{~Hz}$ solenoid valves operating under pulsewidth modulation. Each actuator is controlled by a pair of such valves regulating the air intake and exhaust. A pneumatic multiplexing circuitry allows the same pneumatic controllers to regulate air to three output ports (the Rutgers Master glove or future elbow and knee rehabilitation units). The multipurpose control interface is self configurable, detecting which device is connected to it at any given time. This eliminates the need for reprogramming every time a rehabilitation glove (small, medium, or large) is plugged in. The electronic interface detects which particular glove is connected and loads its "signature" calibration equations automatically, from resident memory.

The host PC is a Pentium $350 \mathrm{MHz}$ which does all the simulation graphics, user mouse/keyboard input, and generates interactive sound. It contains a graphics accelerator board, and an InsideTrack 3D magnetic tracker [12]. This tracker has a small receptor placed on the patient's wrist which measures position and orientation 60 times/s. This function supplements the fingertip position measurement provided by the Rutgers Master glove, and is needed by the host PC for the virtual reality simulation. The microphone array is used to provide hands-free voice commands to the system, by using a voice recognition software running in the background. The camera gives the patient the ability to teleconference with the clinic when needed.

The clinic PC is similar to the home PC, but does not need a graphics accelerator nor the three-dimensional (3-D) tracker. It is used as a server that reads patient's exercise data, stores it in a database, and analyzes the patient's progress. Communication between the home and clinic 

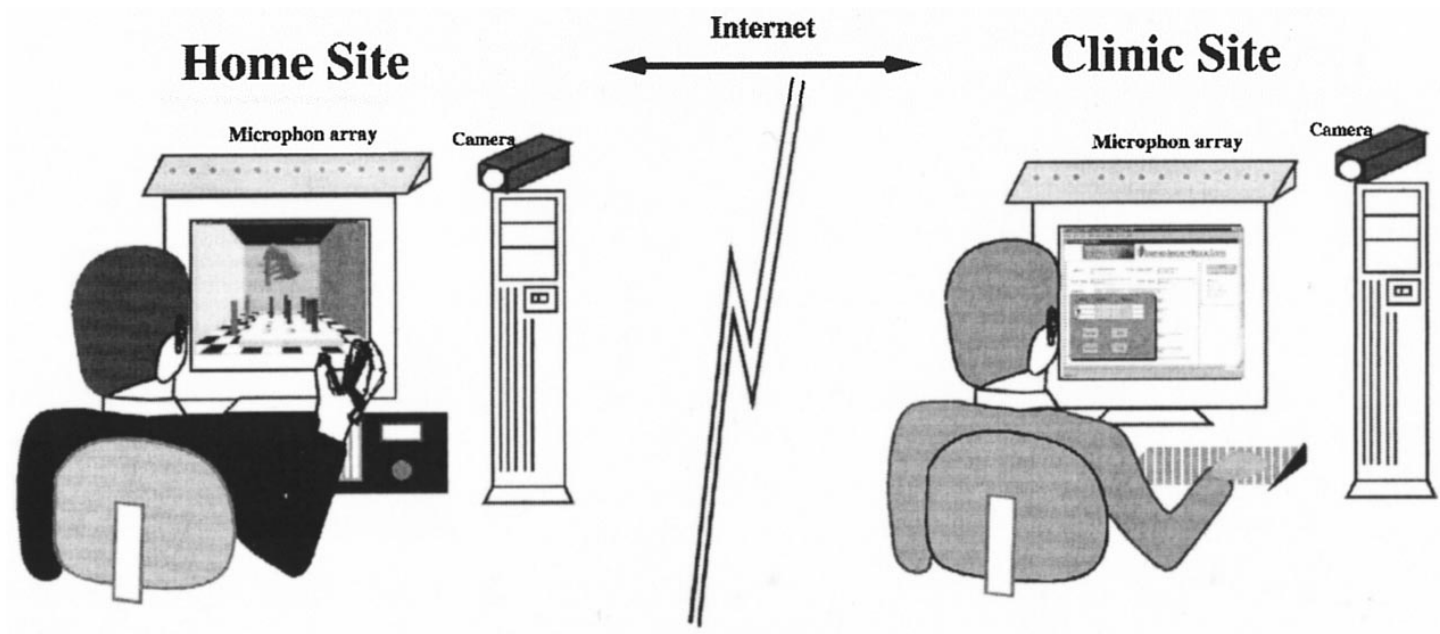

Fig. 1. The telerehabilitation system architecture.

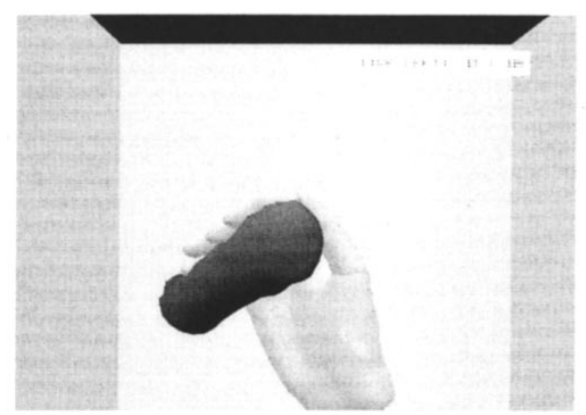

a)

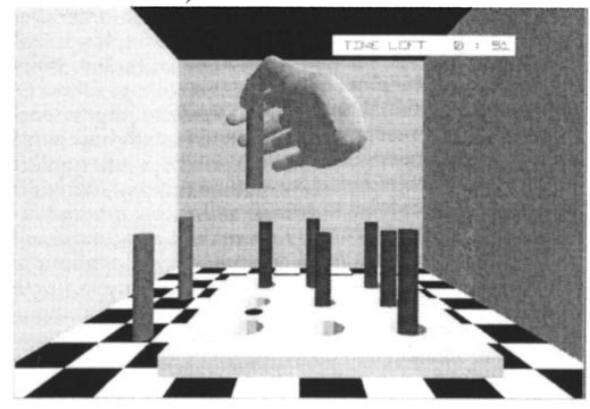

c)

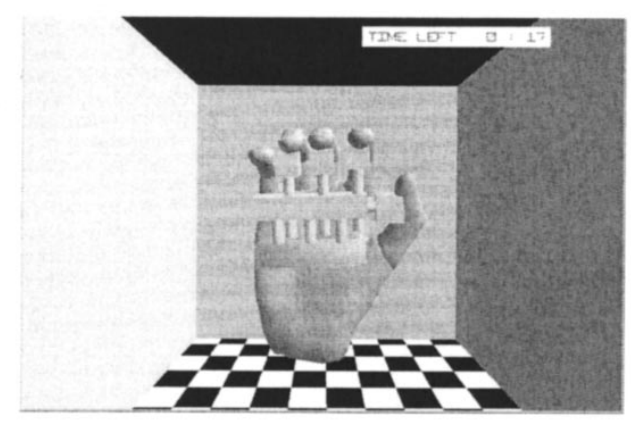

b)

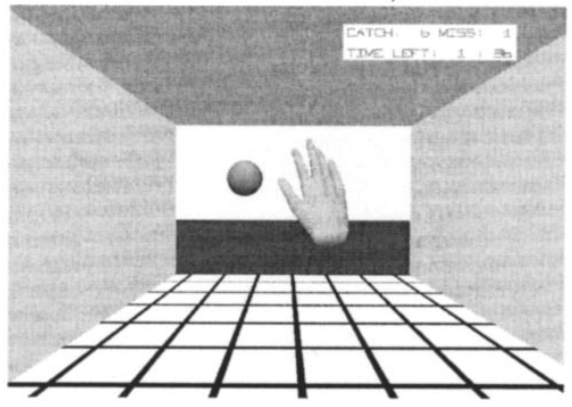

d)

Fig. 2. Virtual rehabilitation exercises. (a) Power putty [13]. (b) DigiKey [4]. (c) Peg board [4]. (d) Hand ball [13]. () The IEEE. Reprinted by permission.

stations is presently done over the Internet/Internet 2, while lower bandwidth telephone lines could also be used.

\section{REHABILITATION SOFTWARE}

The rehabilitation software library has three main components, the 3-D graphics environment, the patient database, and the graphical user interface (GUI). The GUI is the combination of windows and menu buttons that allow a computer novice to operate the system with a mouse. The 3-D graphics library was built using the WorldToolKit commercial software library [5] and contains virtual reality modules [3], each dedicated to a given exercise. Each module currently depicts a virtual hand which mimics the patient's right hand. The hand 3-D model was ported from the Viewpoint DataLabs library, and segmented to allow independent finger motion [15]. The virtual hand interacts with other virtual objects, within an exercise room. The room has a tiled floor for better 3-D perspective, since the image displayed is monoscopic. The overall scene complexity is approximately 1500 polygons, allowing a graphics refresh rate of about 30 frames/s.

The VR simulations are designed for physical rehabilitation and for functional rehabilitation. The physical rehabilitation modules are rubber ball squeezing, the virtual power putty and the virtual DigiKey [see Fig. 2(a), (b)]. These are designed to increase finger force exertion and range of motion. The functional rehabilitation exercises are the ball game and the peg board [Fig. 2(c), (d)]. Each exercise has several levels of difficulty, and has an allowed amount of time. For example, the virtual DigiKey is color-coded (similar to the real one) to indicate maximum force exertion levels. The peg board level of difficulty relates to the peg-hole tolerance, while the ball game has "fast" or "slow" ball velocity levels. 
THUMB
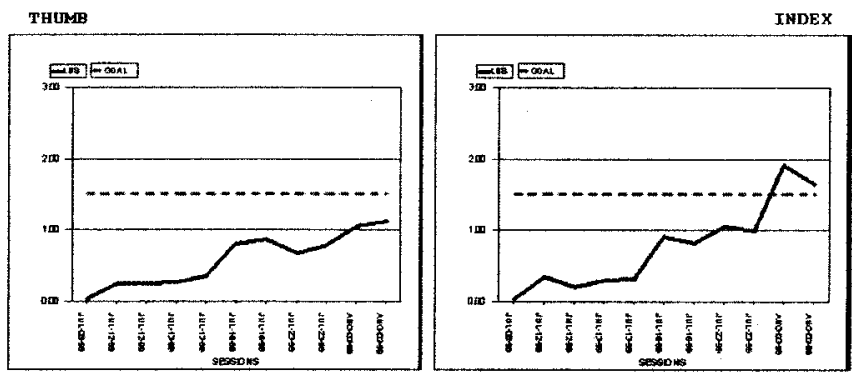

MIDDLE

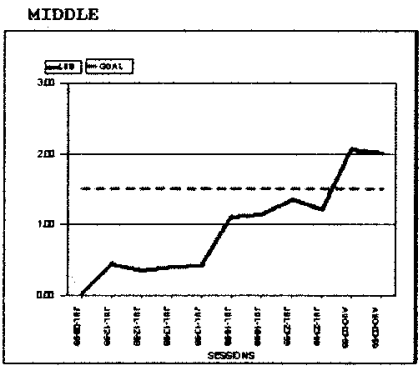

RING

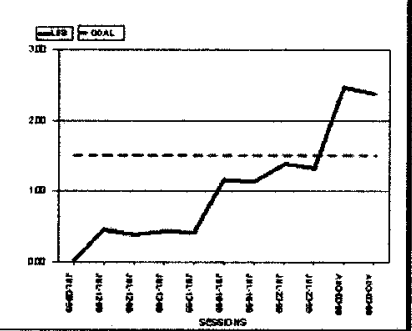

a)

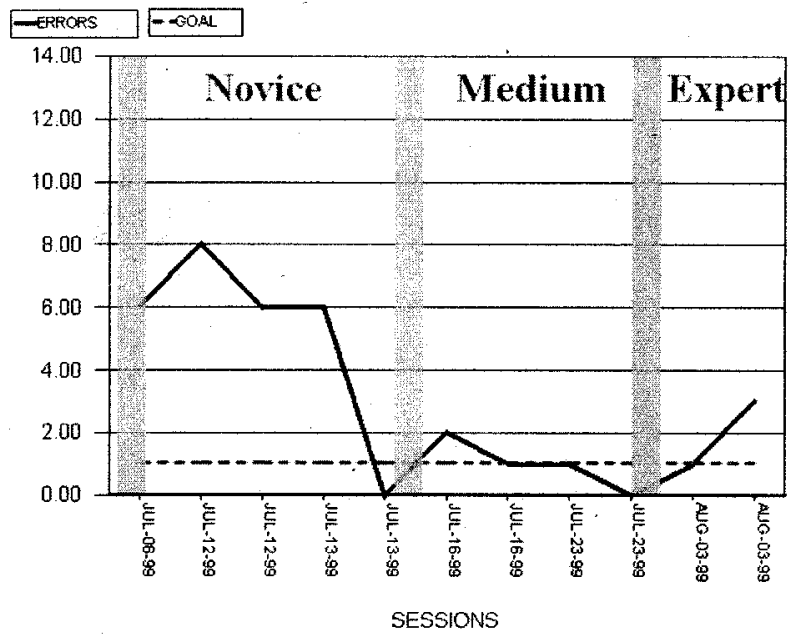

b)
Fig. 3. Patient rehabilitation history. (a) DigiKey. (b) Peg board.

The patient's database was built using the Oracle commercial software [11] and is designed to transparently sample and store all patient data while the exercise is taking place. In the case of hand rehabilitation the data is stored in "low level" and "high level" formats. The low-level data is the time history of finger forces during the exercise, while high-level data consists of the finger-specific average force and effort level (force $\times$ displacement). Yet another level of abstraction is the time history of effort over a series of rehabilitation sessions. A remote therapist accessing the database can easily see how the patient progresses compared to the goal set at the start of therapy. For example, Fig. 3 shows the progress in a patient performing the DigiKey and the peg board exercises. Over a period of two months this patient showed significant increase in finger grasping forces, as well as improved hand-eye coordination function. The patient did not undergo classical clinic-based rehabilitation for his hand impairments. Data was generated on the West Coast (Stanford) and uploaded remotely over the Internet to the East Coast server (Rutgers).

\section{CONCLUSION AND FUTURE WORK}

The telerehabilitation system described above is currently in preclinical trials at the Stanford University Medical School, with remote monitoring from Rutgers University CAIP Center. Validation data for the system will include efficacy in rehabilitation, subjective evaluations by the patients and therapist, and network quality-of-service measurements. The use of virtual reality in telerehabilitation has certain advantages over the classical clinic-based approach. Exercises can be made to look like a game, making therapy fun and motivating to the patient. The ability to store data transparently in real time and to allow remote monitoring gives therapists much more control over patient's rehabilitation routine. At the same time the patient and his/her family is more involved in the rehabilitation process which is taking place at home and at a flexible time. Objective measures of recovery are extracted from the data, and made available on line, reducing the current subjectiveness in patient initial and subsequent evaluations. Eventually one therapist will be able to interface with, and monitor, several remote patients in what we call "multiplexed telerehabilitation."

Future hardware interfaces designed to work with the multi-purpose electronic box are units for the elbow and knee. A haptic interface for the ankle, called the "Rutgers Ankle" [8] has already been built and is presently undergoing pre-clinical trials at the University of Medicine and Dentistry of New Jersey. Large-scale clinical trials will follow once the hardware/software modules have been refined.

\section{REFERENCES}

[1] E. Brandt and A. Pope, Eds., Enabling America-Assessing the Role of Rehabilitation Science and Engineering. Washington, D.C.: National Academy Press, 1997.

[2] G. Burdea, J. Zhuang, E. Roskos, D. Silver, and N. Langrana, "A portable dextrous master with force feedback," in Presence: Teleoperators and Virtual Environments. Cambridge, MA: MIT Press, 1992, vol. 1 , pp. $18-28$.

[3] G. Burdea and P. Coiffet, Virtual Reality Technology. New York: Wiley, 1994.

[4] G. Burdea, S. Deshpande, V. Popescu, N. Langrana, D. Gomez, D. DiPaolo, and M. Kanter, "Computerized hand diagnostic/rehabilitation system using a force feedback glove," Med. Meet Virtual Reality 5, pp. 141-150, January 1997.

[5] World Tool Kit Reference Manual, Engineering Animation, Inc., Feb. 1998.

[6] Electronic Healthcare Systems, Inc., http://dm3host.com/websites $2 /$ ehs/charttrad.html..

[7] S. Fox, "EVAL-revolutionizing hand exams," ADVANCE for Occupational Therapists, vol. 7, no. 3, p. 7, 1991.

[8] M. Girone, G. Burdea, M. Bouzit, V. Popescu, and J. E. Deutsch, "Orthopedic rehabilitation using the 'Rutgers Ankle' interface," in Proc. Virtual Reality Meets Med. 2000. Amsterdam, The Netherlands: IOS Press, 2000, vol. 70, pp. 89-95.

[9] Greenleaf Medical Systems. (1997) Business Overview, Palo Alto, CA [Online]. Available: http://www.greenleafmed.com/Products/pointofcare.html.

[10] Lafayette Instrument Company. [Online]. Available: http://www.licmef.com/assessme1.htm

[11] Oracle User's Manual. Redwood City, CA: Oracle, Co., 1995.

[12] 3Space InsideTrack HP User's Manual. Colchester, VT: Polhemus, Co., 1997.

[13] V. Popescu, G. Burdea, M. Bouzit, M. Girone, and V. Hentz, "PC-based telerehabilitation system with force feedback," in Proc. Med. Meets Virtual Reality (7) Conf., vol. 62. Amsterdam, The Netherlands, 1999, pp. 262-267.

[14] V. Popescu, G. Burdea, M. Bouzit, M. Girone, and V. Hentz, "Orthopedic telerehabilitation with virtual force feedback," IEEE Trans. Inform. Technol. Biomed., vol. 4, pp. 45-51, Mar. 2000.

[15] Viewpoint DataLabs International, , Viewpoint Catalog, Third ed. Orem, UT, 1994. 